\title{
Short Note \\ Green's Functions for Surface Waves in a Generic Velocity Structure
}

\author{
by Victor C. Tsai and Sarun Atiganyanun*
}

\begin{abstract}
Methodologies for calculating surface-wave velocities and the associated displacement/stress eigenfunctions and Green's functions have been well established for many decades. However, to our knowledge, no one has ever documented a quantitative evaluation of these properties for commonly used empirical scalings. For example, it is currently not possible to take a given power-law dependence of shear-wave velocity on depth and look up the corresponding dependence of phase velocity on frequency, or Green's function surface displacement. We address this gap in the literature and here provide explicit quantitatively accurate expressions for phase velocities and Green's function amplitudes for a few commonly used empirical formulas for near-surface velocity structure. These exact expressions are found to be immediately useful in applications that use shallow phase velocities and also in applications that interpret seismic amplitudes or amplitude ratios from near-surface processes such as fluvial transport, icequakes, landslides, and volcanic tremor.
\end{abstract}

\section{Introduction}

In the last decade, there has been an increasing interest in using seismology to quantitatively analyze near-surface processes such as landslides (e.g., Ekstrom and Stark, 2013), glaciers (e.g., Tsai and Ekstrom, 2007), rivers (e.g., Burtin et al., 2008), and volcanic tremor (e.g., McNutt and Nishimura, 2008). In parallel, there has also been an exponential growth in ambient noise cross-correlation applications in which a given station-pair cross correlation is assumed to be related to the surface-wave Green's function (e.g., Snieder, 2004; Shapiro et al., 2005). In both cases, these studies either explicitly or implicitly rely on knowing the surface-to-surface Green's function (or impulse response function) to make quantitative use of data.

However, despite such surface-to-surface Green's functions being necessary, an explicit form for these Green's functions is rarely described in detail, with many authors assuming their readers have access to the numerical software necessary to reproduce the Green's functions. Although this may be a fair assumption in many cases, it is not in others. Moreover, calculating the complete Green's functions for a specific case may take significant time, whereas the reader is often interested in an approximate but quantitative estimate of the Green's function. Methodologies for computing Green's functions have been around for many decades (e.g., Alterman et al., 1959), but it still is not possible for a reader to perform the quick quantitative estimate described above. In this short note, we address this problem by providing explicit results for

*Now at the Department of Nanoscience and Microsystems, University of New Mexico, Albuquerque, New Mexico 87131.
Love- and Rayleigh-wave Green's functions for some generic seismic structures. It is expected that these quantitative results will be useful for the study of noise cross correlations as well as near-surface processes.

\section{Methodology}

The goal of this short note is to quantitatively derive expressions for surface-wave (both Love and Rayleigh) phase velocities and Green's function amplitudes for a few simple given velocity structures, for which it is assumed that shear modulus $\mu(z)$, Poisson's ratio $\nu(z)$, and density $\rho(z)$ are all provided as a function of depth $z$. To accomplish this, we follow the numerical integration approach of Alterman et al. (1959), as described in detail in Aki and Richards (2002, pp. 267-268). For each given frequency, $\omega=2 \pi f$, one solves for $k=2 \pi / \lambda$, along with the associated eigenfunctions (displacement $l_{1}(z)$ and stress $l_{2}(z)$ for Love waves; displacements $r_{1}(z)$ and $r_{2}(z)$ and stresses $r_{3}(z)$ and $r_{4}(z)$ for Rayleigh waves). Once these are known, phase velocities are given by $c(\omega)=\omega / k(\omega)$ and the frequency-domain far-field Green's functions can be written as

$$
\begin{aligned}
G^{L}(\omega)= & \frac{l_{1}(z) l_{1}(h)}{8 c_{L} U_{L} I_{1}^{L}} \sqrt{\frac{2}{\pi k_{L} r}} e^{i\left(k_{L} r+\frac{\pi}{4}\right)} \\
& \times\left[\begin{array}{ccc}
\sin ^{2} \phi & -\sin \phi \cos \phi & 0 \\
-\sin \phi \cos \phi & \cos ^{2} \phi & 0 \\
0 & 0 & 0
\end{array}\right]
\end{aligned}
$$


and

$$
G^{R}(\omega)=\frac{e^{i\left(k_{R} r+\frac{\pi}{4}\right)}}{8 c_{R} U_{R} I_{1}^{R}} \sqrt{\frac{2}{\pi k_{R} r}}\left[\begin{array}{ccc}
r_{1}(z) r_{1}(h) \cos ^{2} \phi & r_{1}(z) r_{1}(h) \sin \phi \cos \phi & -i r_{1}(z) r_{2}(h) \cos \phi \\
r_{1}(z) r_{1}(h) \sin \phi \cos \phi & r_{1}(z) r_{1}(h) \sin ^{2} \phi & -i r_{1}(z) r_{2}(h) \sin \phi \\
i r_{2}(z) r_{1}(h) \cos \phi & i r_{2}(z) r_{1}(h) \sin \phi & r_{2}(z) r_{2}(h)
\end{array}\right],
$$

in which $G_{x y}^{L}(w)$ and $G_{x y}^{R}(w)$ are the Love and Rayleigh displacements in the $x$ direction for a unit sinusoidal load $e^{-i \omega t}$ in the $y$ direction, and $k_{L}(\omega)$ and $k_{R}(\omega)$ are the Love and Rayleigh wavenumbers, respectively (Aki and Richards, 2002). $c_{p}$, $U_{p}$, and $I_{1}^{p}$ are phase velocity, group velocity, and integrals of the eigenfunctions over depth, for Love $(p=L)$ and Rayleigh waves $(p=R)$. Note that $c_{p}=\omega / k_{p}(\omega)$, $U_{p}=\left(\partial k_{p} / \partial \omega\right)^{-1}, \quad 2 I_{1}^{L}=\int_{0}^{\infty} \rho(z) l_{1}(z)^{2} d z, \quad$ and $2 I_{1}^{R}=$ $\int_{0}^{\infty} \rho(z)\left[r_{1}(z)^{2}+r_{2}(z)^{2}\right] d z$.

In this work, we concentrate on near-surface forces and observations for which surface waves are expected to dominate, and therefore we begin by setting $z=h=0$ in the Green's functions. As discussed in Tsai et al. (2012), the factor $r_{2}^{2}(0) / I_{1}^{R}$ scales with $k_{R} / \rho_{0}$ (for constant density $\rho_{0}$ ), and we expect similar scalings for $l_{1}^{2}(0) / I_{1}^{L}$ and $r_{i}(0) r_{j}(0) / I_{1}^{R}$. With known $k_{p}(\omega)$, the surface-to-surface Green's function is therefore completely characterized by one and two nondimensional numbers for Love and Rayleigh waves, respectively, as

$$
N^{L}=\frac{\rho(0) l_{1}^{2}(0)}{k_{L} I_{1}^{L}} \quad \text { and } \quad N_{i j}^{R}=\frac{\rho(0) r_{i}(0) r_{j}(0)}{k_{R} I_{1}^{R}},
$$

in which $i$ and $j$ are either 1 or 2 , and it is easily shown that $N_{12}^{R}=N_{21}^{R}$ and $\left(N_{12}^{R}\right)^{2}=N_{11}^{R} N_{22}^{R}$ (leaving only two independent numbers, say $N_{11}^{R}$ and $N_{12}^{R}$ for Rayleigh waves). The depth dependence of the Green's function will be discussed later. The amplitude of the surface-to-surface Green's functions can then be written as

$$
\begin{aligned}
\left|G^{L}\right| & =\frac{N^{L} k_{L}}{8 \rho_{0} c_{L} U_{L}} \sqrt{\frac{2}{\pi k_{L} r}} e^{-\omega r /\left(2 U_{L} Q_{L}\right)} \\
\text { and } \quad\left|G_{i j}^{R}\right| & =\frac{N_{i j}^{R} k_{R}}{8 \rho_{0} c_{R} U_{R}} \sqrt{\frac{2}{\pi k_{R} r}} e^{-\omega r /\left(2 U_{R} Q_{R}\right)},
\end{aligned}
$$

in which the directional dependences are as in equations (1a) and (1b), $\rho_{0}=\rho(0)$, and we have added anelastic terms that depend on $Q_{p}$ (where $p=L$ or $R$, as before). Here, $G_{i j}^{R}$ denotes only the two inplane components of the Rayleigh wave, with $j$ being either a longitudinal or vertical force ( 1 and 2 , respectively), and $i$ being the longitudinal or vertical displacement directions. The full $G^{R}$ is easily constructed from $G_{i j}^{R}$ from analogy to equation (1b).

\section{Results}

We tabulate results for a few different examples of empirical scalings that have been proposed recently. At first, we focus on a class of power-law scalings in which shear veloc- ity is a power-law function of depth, $\beta(z)=\beta_{0}\left(z / z_{0}\right)^{\alpha}$, and in which density is constant, $\rho(z)=\rho_{0}$. This class of powerlaw scalings includes one of the simple scalings of Boore and Joyner (1997), and many studies of near-surface structure use generic models that are well fit by power-law scalings (e.g., Wald et al., 1991; Silva et al., 1999). As will be shown, this power-law velocity structure also results in a power-law dependence of phase velocity on frequency, which has been a common assumption of various authors (e.g., Ferrazzini et al., 1991; Metaxian et al., 1997). Other empirical analysis has also used piecewise power-law scalings (and with density scaling with velocity), and results for such a case will be discussed later. In this study, we only calculate results for fundamental mode $(n=0)$ surface waves due to their usual observed dominance, but the same analysis could be extended to examine surface-wave overtones.

\section{Power-Law Scaling of Velocity and Constant Density}

With power-law scaling of shear velocity, $\beta(z)=$ $\beta_{0}\left(z / z_{0}\right)^{\alpha}$, constant Poisson's ratio $v$ and constant density $\rho_{0}$, nondimensionalization of the governing equations is found to be particularly enlightening. Choosing length scale $z_{0}$ and velocity scale $\beta_{0}$, then $l_{1}^{\prime}=l_{1} / z_{0}$ and similarly for $r_{1}$ and $r_{2}$, and $l_{2}^{\prime}=l_{2} / \rho_{0} \beta_{0}^{2}$ and similarly for $r_{3}$ and $r_{4}$. This also leads to $z^{\prime}=z / z_{0}, k_{p}^{\prime}=z_{0} k_{p}, \omega^{\prime}=z_{0} \omega / \beta_{0}$, and governing equations that are independent of dimensional parameters. (The Rayleigh-wave system still depends on Poisson's ratio.) For example, the Love-wave governing equations can then be written as

$$
\frac{d}{d z^{\prime}}\left[\begin{array}{l}
l_{1}^{\prime} \\
l_{2}^{\prime}
\end{array}\right]=\left[\begin{array}{cc}
0 & \left(z^{\prime}\right)^{-2 \alpha} \\
\left(k^{\prime}\right)^{2}\left(z^{\prime}\right)^{2 \alpha}-\left(\omega^{\prime}\right)^{2} & 0
\end{array}\right]\left[\begin{array}{l}
l_{1}^{\prime} \\
l_{2}^{\prime}
\end{array}\right] .
$$

In terms of the nondimensional solution $k_{p}^{\prime}\left(\omega^{\prime}\right)$, the dimensionally correct frequency and phase velocity are therefore given by $\omega=\beta_{0} \omega^{\prime} / z_{0}$ and $c_{p}=\omega / k_{p}=\beta_{0} \omega^{\prime} / k_{p}^{\prime}$. At this point, one can observe that for the power-law scaling of velocity, the ratio $\gamma_{0} \equiv \beta_{0} / z_{0}^{\alpha}$ (along with $\alpha$ ) completely determines $\beta$ so that $\beta_{0}$ and $z_{0}$ are not independent. For a given $\gamma_{0}$ and $\alpha$, $\beta_{0}$ is related to $z_{0}$ by $\beta_{0}=\gamma_{0} z_{0}^{\alpha}$. Perhaps surprisingly, this implies that the solution for $k_{p}^{\prime}$ for a single choice of $\omega^{\prime}$ (e.g., $\omega^{\prime}=1$ ) completely determines the behavior of $c_{p}$ for all $\omega$ because one can rewrite $\omega=\gamma_{0} \omega^{\prime} z_{0}^{\alpha-1}$ and therefore

$$
\begin{aligned}
c_{p}(\omega) & =\frac{\omega}{k_{p}}=\frac{\beta_{0} \omega^{\prime}}{k_{p}^{\prime}}=\frac{\gamma_{0} \omega^{\prime}}{k_{p}^{\prime}} z_{0}^{\alpha}=\frac{\gamma_{0} \omega^{\prime}}{k_{p}^{\prime}}\left(\frac{\gamma_{0} \omega^{\prime}}{\omega}\right)^{\frac{\alpha}{1-\alpha}} \\
& =\frac{\left(\gamma_{0} \omega^{\prime}\right)^{1 /(1-\alpha)}}{k_{p}^{\prime}} \omega^{-\frac{\alpha}{1-\alpha}}=c_{p 0}\left(\frac{\omega}{\omega_{0}}\right)^{-\frac{\alpha}{1-\alpha}} .
\end{aligned}
$$




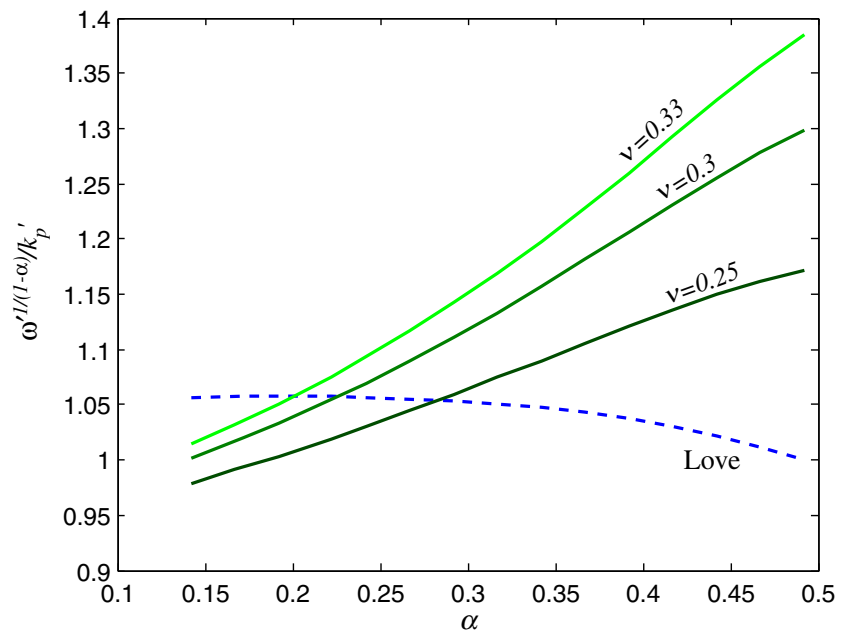

Figure 1. Nondimensional phase velocity coefficient $\left(\omega^{\prime}\right)^{1 /(1-\alpha)} / k_{p}^{\prime}$ for Love waves (dashed line) and Rayleigh waves (solid lines) for a range of power-law exponents $\alpha$. Rayleigh results are plotted for three choices of Poisson's ratio $(0.25,0.3$, and 0.33 , with shading going from dark to light). As explained in the text, $c_{p 0}$ is obtained by multiplying the nondimensional coefficient by $\left[\beta_{0} /\left(\omega_{0} z_{0}\right)^{\alpha}\right]^{1 /(1-\alpha)}$; see equation (5) for details, including phase velocity scaling with frequency. These exact results differ from the approximate scaling of Tsai et al. (2012) by only a modest factor that is independent of all parameters (including frequency $\omega$ ) except $\alpha$. The approximate result of Tsai et al. (2012) can therefore also be expressed as a nondimensional phase velocity coefficient with a value of $\Gamma(1+\alpha)^{1 /(1-\alpha)}$, in which $\Gamma(x)$ is the gamma function. The color version of this figure is available only in the electronic edition.

Similarly, $l_{1}^{\prime}\left(\omega^{\prime}\right)$ for a single choice of $\omega^{\prime}$ completely determines $l_{1}(\omega)$. Moreover, nondimensionalization of equation (2) shows that $N^{L}$ and $N_{i j}^{R}$ are actually independent of $\omega$. For example, $N^{L}=2\left(l_{1}^{\prime}\right)^{2}(0) / k_{L}^{\prime} \int_{0}^{\infty}\left(l_{1}^{\prime}\right)^{2}\left(z^{\prime}\right) d z^{\prime}$, which is similar to that for $N_{i j}^{R}$. Because the governing equations, like equation (4), are also independent of dimensional parameters, $k_{p}^{\prime}, N^{L}$, and $N_{i j}^{R}$ are all independent of $\beta_{0}$ (or $\gamma_{0}$ ) and only depend on $\alpha$ (and $\nu$ for Rayleigh waves). This exact result confirms that the power-law scaling of velocity with depth implies a power-law scaling of phase velocity with frequency, as was suggested by the approximate analysis of Tsai et al. (2012) (in which eigenfunctions were assumed to decay exponentially with depth), as well as that of Godin and Chapman (2001). The result additionally indicates that the complete frequency dependence is determined by a single constant for phase velocity and single constants for $N^{L}$ and $N_{i j}^{R}$.

In Figure 1, we plot the constant $\left(\omega^{\prime}\right)^{1 /(1-\alpha)} / k_{p}^{\prime}$ (normalized phase velocity) for a range of reasonable values of $\alpha$ (e.g., Boore and Joyner, 1997) and, in Figure 2, we plot $N^{L}$ and $N_{i j}^{R}$ (normalized amplitudes) for the same range of $\alpha$ and three different values of Poisson's ratio $(\nu=0.3,0.25$, and 0.33 in Fig. 2a, 2b, and 2c, respectively). The values in Figure 2 can be directly substituted into equation (3) to obtain the Green's function amplitudes. It is of interest that the relative
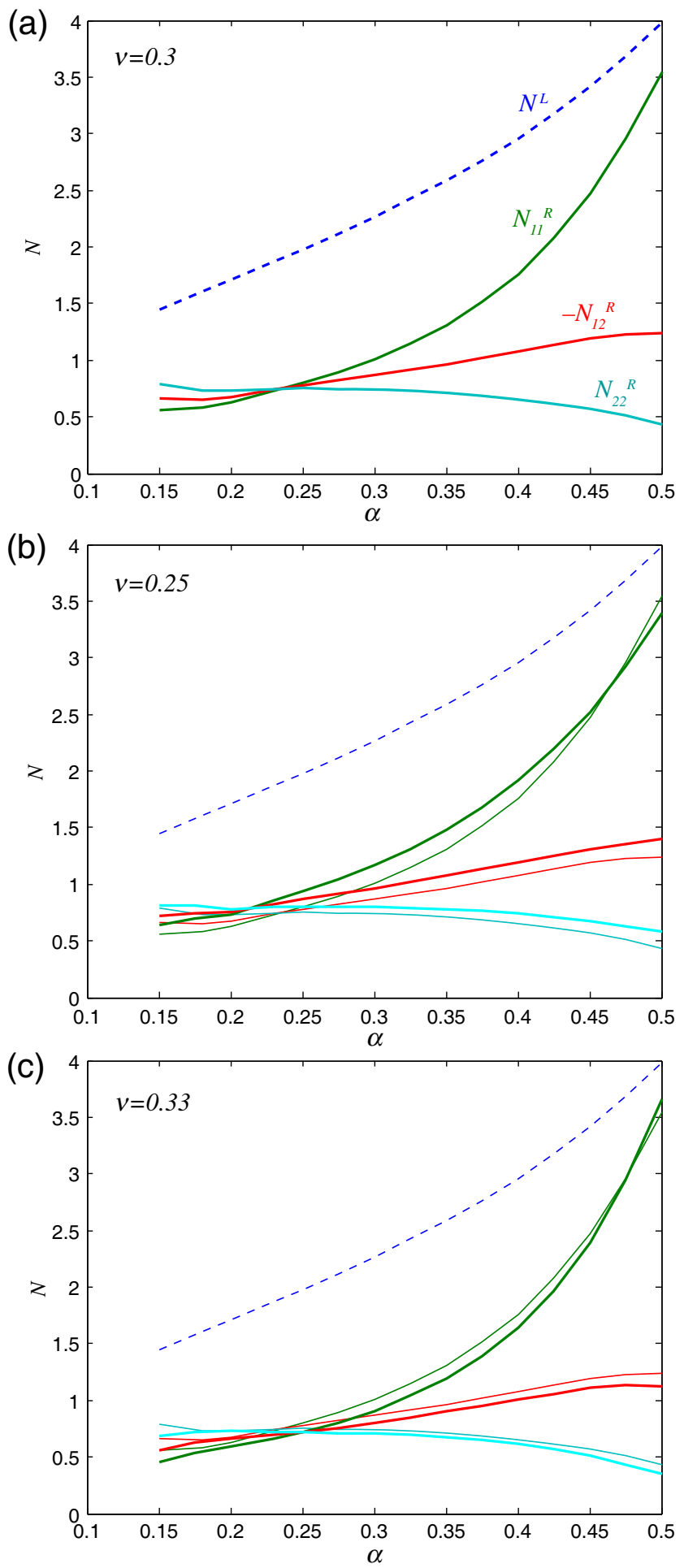

Figure 2. Nondimensional Green's function amplitudes $N^{L}$ and $N_{i j}^{R}$, as described in equation (2), for a range of power-law exponents $\alpha$. In each panel, shading is identical and corresponds to the labels in panel (a). Note that $-N_{12}^{R}$ is the geometric mean of $N_{11}^{R}$ and $N_{22}^{R}$, and $N_{12}^{R} / N_{22}^{R}$ is commonly referred to as the Rayleigh-wave $\mathrm{H} / \mathrm{V}$ ratio or Rayleigh-wave ellipticity. The results are shown for (a) the default Poisson's ratio of $\nu=0.3$; (b) $\nu=0.25$ (thick lines), with results in (a) as thin lines for reference; and (c) $\nu=0.33$ (thick lines), with results in (a) as thin lines for reference. The color version of this figure is available only in the electronic edition. 

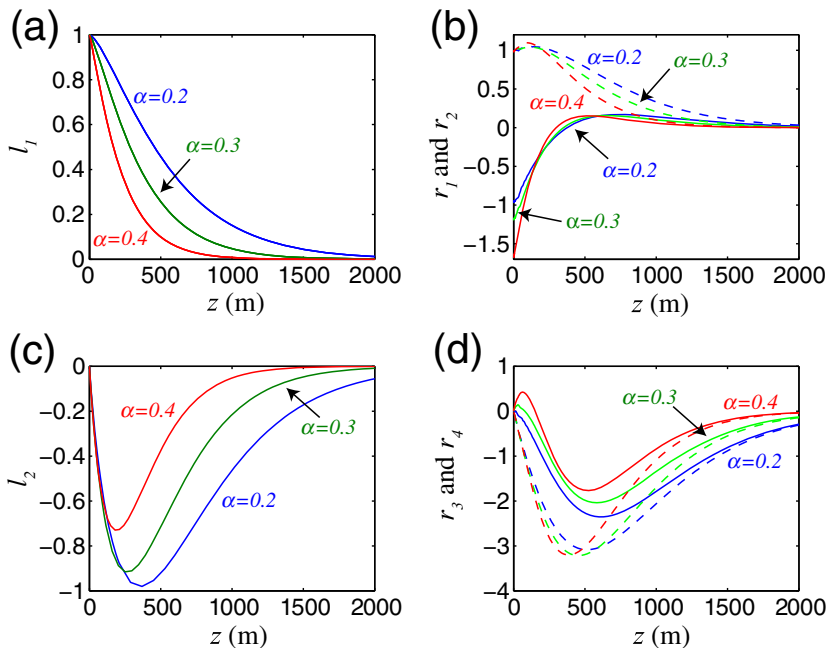

Figure 3. Eigenfunctions $l_{i}$ and $r_{i}$ versus depth for $f=1 \mathrm{~Hz}$ : (a) $l_{1}(z)$ for $\alpha=0.2,0.3$, and 0.4 , as labeled, normalized by $l_{1}(0)$; (b) $r_{1}(z)$ (solid line; horizontal displacement) and $r_{2}(z)$ (dashed; vertical displacement), normalized by $r_{2}(0)$; (c) $l_{2}(z)$; and (d) $r_{3}(z)$ (solid line) and $r_{4}(z)$ (dashed line). Shading in (b)(d) are as in (a). The color version of this figure is available only in the electronic edition.

Love-wave amplitudes increase with $\alpha$, as might be expected with higher confinement of energy at shallower depths. In addition, the ratio of horizontal-to-vertical $(\mathrm{H} / \mathrm{V})$ Rayleighwave energy (e.g., $N_{11}^{R} / N_{22}^{R}$ ) also increases with $\alpha$, for similar reasons, as is well known from microtremor studies (e.g., Fah et al., 2001). To obtain Love- and Rayleigh-wave phase velocities, one should take the values in Figure 1 and multiply by $\left[\beta_{0} /\left(\omega_{0} z_{0}\right)^{\alpha}\right]^{1 /(1-\alpha)}$ to obtain $c_{p 0}$. For example, for the empirical shear-wave profile of Boore and Joyner (1997), in which $\beta_{0}=2206 \mathrm{~m} / \mathrm{s}, z_{0}=1000 \mathrm{~m}$, and $\alpha=0.272$, the factor in Figure 1 is 1.06 for Love waves and 1.09 for Rayleigh waves $(\nu=0.3)$ so that $c_{L}=1582 \mathrm{~m} / \mathrm{s}(f / 1 \mathrm{~Hz})^{-0.374}$ and $c_{R}=1626 \mathrm{~m} / \mathrm{s} \quad(f / 1 \mathrm{~Hz})^{-0.374}$. For this same profile, $N^{L}=2.10, N_{11}^{R}=0.88, N_{12}^{R}=-0.81$, and $N_{22}^{R}=0.75$, so that the Rayleigh-wave $\mathrm{H} / \mathrm{V}$ amplitude ratio is 1.08 . It is also of interest that the Rayleigh-wave $\mathrm{H} / \mathrm{V}$ ratio transitions from less than 1 to greater than 1 as $\alpha$ increases above 0.234 for $\nu=0.3,0.213$ for $\nu=0.25$, and 0.249 for $\nu=0.33$. For the majority of near-surface structures $\alpha>0.23$, implying Rayleigh-wave $\mathrm{H} / \mathrm{V}$ ratios are typically greater than 1 , as expected. Figure 3 shows the eigenfunctions $l_{i}$ and $r_{i}$ for a few different choices of $\alpha(0.2,0.3$, and 0.4$)$.

\section{Piecewise Power-Law Scaling of Velocity}

To supplement the results for a simple power-law scaling of velocity with depth, we provide one example of a more realistic generic rock site velocity profile from Boore and Joyner (1997) which is composed of five separate piecewise power-law scalings that are joined together as

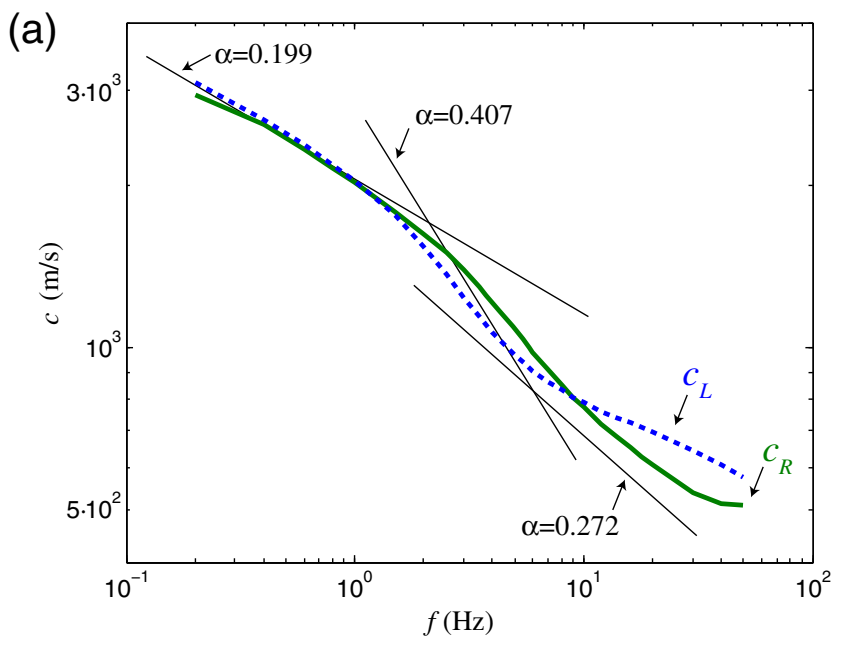

(b)

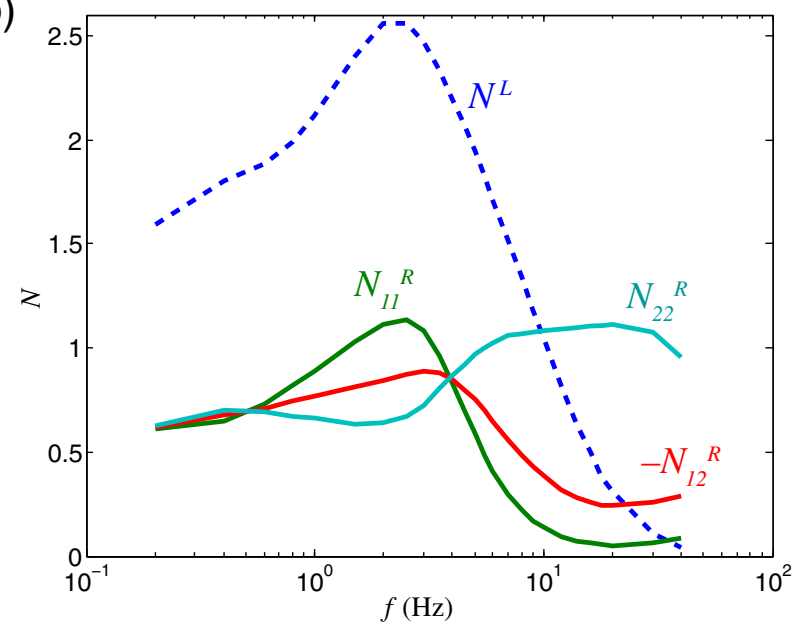

Figure 4. Phase velocities and nondimensional Green's function amplitudes for the piecewise power-law scaling for $\beta$ and $\rho(\beta)$ of Boore and Joyner (1997) and $\nu=0.3$. (a) Phase velocity versus frequency $f=\omega / 2 \pi$ for Love waves (dashed line) and Rayleigh waves (solid line). Thin lines are the exact power-law results (including prefactor) for the individual segments at a given $\alpha$ (as noted), as from equation (6). (b) Amplitudes $N^{L}$ and $N_{i j}^{R}$ versus frequency $f$. Shading is the same as in Figure 2. The color version of this figure is available only in the electronic edition.

$$
\beta(z)= \begin{cases}245, & z^{\prime} \leq 0.001 \\ 2206\left(z^{\prime}\right)^{0.272}, & 0.001<z^{\prime} \leq 0.030 \\ 3542\left(z^{\prime}\right)^{0.407}, & 0.030<z^{\prime} \leq 0.190 \\ 2505\left(z^{\prime}\right)^{0.199}, & 0.190<z^{\prime} \leq 4.00 \\ 2927\left(z^{\prime}\right)^{0.086}, & 4.00<z^{\prime}\end{cases}
$$

in which $z^{\prime}=z /(1000 \mathrm{~m})$ and $\beta$ is in meters/second. This piecewise power law is thought to be more realistic than the single power law over a wide range of depths but has the obvious disadvantage that it cannot be treated in the same way as our previous analysis for a single power law. Instead, we must perform computations over a range of frequencies, and in Figure 4 we show results for frequencies ranging from 0.2 to $40 \mathrm{~Hz}$ (with $\nu=0.3$ ). Here we also use the more 
realistic empirical scaling of density with shear velocity of $\rho=2500+93.75 \times(\beta / 1 \mathrm{~km} / \mathrm{s}-0.3)$, in which $\rho$ is in kilograms per cubic meter (Boore and Joyner, 1997). This empirical scaling of density is only modestly different from a constant, and it may be noted that using a constant density yields similar results.

As shown in Figure 4a, both Love- and Rayleigh-wave phase velocities no longer follow pure power-law trends but approximately follow the expected trends (see thin black lines of Fig. 4a) over some limited range of frequencies and with sometimes substantially different prefactor constants. It is worth noting that the frequencies at which the different power-law trends transition occur at roughly those expected based on the depth sensitivity of waves of a given frequency. For example, the transition from $\alpha=0.199$ to $\alpha=0.407$ occurs close to $2 \mathrm{~Hz}$, at which $1 / k_{L} \sim 120 \mathrm{~m}$ and $1 / k_{R} \sim 130 \mathrm{~m}$, close to the $190 \mathrm{~m}$ depth at which the assumed velocity structure changes power-law form.

On the other hand, although the scaled Green's function amplitudes $N^{L}$ and $N_{i j}^{R}$ (Fig. 4b) have the expected behavior of large $N^{L}$ and $N_{11}^{R} / N_{22}^{R}$ (H/V ratio) for higher $\alpha$ (and thus more pronounced of a low-velocity layer), the transitions are at much lower frequencies than those for phase velocities. For example, the $\mathrm{H} / \mathrm{V}$ ratio transitions from less than 1 to greater than 1 at roughly 0.5 and $4 \mathrm{~Hz}$, respectively, where the naive predictions based on depth sensitivity would put these transitions at roughly 2 and $50 \mathrm{~Hz}$, respectively. This general feature that Rayleigh-wave $\mathrm{H} / \mathrm{V}$ ratios are sensitive to much shallower structure than might be intuitively guessed agrees well with the previous results of Lin et al. (2012) for longer periods. Although not entirely intuitive, the primary reason for this behavior is that the boundary conditions are much more strongly affected by near-surface velocity structure than the average shape of the eigenfunctions $l_{i}$ and $r_{i}$. We also note that the peak in $N^{L}$ coincides nearly identically with the peak of $N_{11}^{R} / N_{22}^{R}$. This may partly explain the difficulty, of traditional microtremor $\mathrm{H} / \mathrm{V}$ studies, from distinguishing between the effects of Rayleigh-wave ellipticity and Loveto-Rayleigh in causing observed microtremor $\mathrm{H} / \mathrm{V}$ ratio differences (Nakamura, 1989; Fah et al., 2001; BonnefoyClaudet et al., 2006).

\section{Conclusions}

In this work, we have evaluated the Love-wave and Rayleigh-wave phase velocities and Green's function amplitudes for a few different commonly used empirical scalings. For a pure power-law dependence of shear-wave velocity on depth and constant density and Poisson's ratio, we find a particularly simple solution, in which results for all frequencies $\omega$ and all $\beta_{0}$ are determined with a single solve of the governing equations for a given frequency $\omega_{0}$. The power-law scaling of phase velocities with frequency is found to agree with the scaling derived by Tsai et al. (2012), but the numerical prefactors are determined quantitatively here. In addition to the pure power-law case, we also evaluate results for the more realistic but generic velocity profile of Boore and Joyner (1997). These results suggest that the simple power-law scalings can be used to understand the results in the entire range of frequencies but that the frequency at which the scalings transition is substantially lower for the Green's function amplitudes, compared with the corresponding transition for phase velocities.

Despite a pure power-law velocity structure (with constant density) being an idealization of any realistic structure, the exact results provided here for this case should prove useful as a first approximation for many surface-wave studies. In particular, the expressions derived are of use for any studies that use near-surface Green's functions. For example, a wide range of ambient noise cross-correlation studies make use of surface-to-surface Green's functions, either explicitly or implicitly (e.g., Shapiro et al., 2005; Lin et al., 2008; Prieto and Beroza, 2008). Other studies of near-surface phenomena such as river sediment transport (Burtin et al., 2008; Tsai et al., 2012), sea ice (Kedar et al., 2008; Stutzmann et al., 2009; Tsai and McNamara, 2011), landslides (Hibert et al., 2011; Ekstrom and Stark, 2013), and volcanic tremor (McNutt and Nishimura, 2008) also make use of surface-to-surface surfacewave amplitudes. For such studies of near-surface seismic sources, the tabulation of the coefficients $N^{L}$ and $N_{i j}^{R}$ will provide a useful estimate of the expected ground-motion amplitudes, which is a first step to utilize the seismic data to constrain physical processes. Although it is always possible to perform a detailed numerical study of wave propagation that will yield more precise results in any particular case, the general results presented here are thought to be useful nonetheless, due in part to their approximate applicability to a wide range of scenarios and also to their ease of use, without needing to perform another numerical simulation.

\section{Data and Resources}

No data were used in this paper.

\section{Acknowledgments}

The authors thank M. Haney and an anonymous reviewer for constructive comments, and the Summer Undergraduate Research Fellowship Program at Caltech and J. Edward Richter for funding S.A.

\section{References}

Aki, K., and P. G. Richards (2002). Quantitative Seismology: Theory and Methods, Second Ed., University Science Books, Sausalito, California. Alterman, Z., H. Jarosch, and C. L. Pekeris (1959). Oscillations of the Earth, Proc. R. Soc. London A, Math. Phys. Sci. 252, 80-95.

Bonnefoy-Claudet, S., F. Cotton, and P.-Y. Bard (2006). The nature of noise wavefield and its applications for site effects studies, Earth Sci. Rev. 79, 205-227.

Boore, D. M., and W. B. Joyner (1997). Site amplifications for generic rock sites, Bull. Seismol. Soc. Am. 87, 327-341.

Burtin, A., L. Bollinger, J. Vergne, R. Cattin, and J. L. Nabelek (2008). Spectral analysis of seismic noise induced by rivers: A new tool to 
monitor spatiotemporal changes in stream hydrodynamics, J. Geophys. Res. 113, no. B05301, doi: 10.1029/2007JB005034.

Ekstrom, G., and C. P. Stark (2013). Simple scaling of catastrophic landslide dynamics, Science 339, 1416-1419.

Fah, D., F. Kind, and D. Giardini (2001). A theoretical investigation of average H/V ratios, Geophys. J. Int. 145, 535-549.

Ferrazzini, V., K. Aki, and B. Chouet (1991). Characteristics of seismic waves composing Hawaiian volcanic tremor and gas-piston events observed by a near-source array, J. Geophys. Res. 96, 6199-6209.

Godin, O. A., and D. M. F. Chapman (2001). Dispersion of interface waves in sediments with power-law shear speed profiles. I. Exact and approximate analytical results, J. Acoust. Soc. Am. 110, 1890-1907.

Hibert, C., A. Mangeney, G. Grandjean, and N. M. Shapiro (2011). Slope instabilities in Dolomieu crater, Reunion Island: From seismic signals to rockfall characteristics, J. Geophys. Res. 116, F04032, doi: 10.1029/ 2011JF002038.

Kedar, S., M. Longuet-Higgins, F. Webb, N. Graham, R. Clayton, and C. Jones (2008). The origin of deep ocean microseisms in the North Atlantic Ocean, Proc. R. Soc. London A, Phys. Eng. Sci. 464, 777-793.

Lin, F.-C., M. P. Moschetti, and M. H. Ritzwoller (2008). Surface wave tomography of the western United States from ambient seismic noise: Rayleigh and Love wave phase velocity maps, Geophys. J. Int. 173, 281-298.

Lin, F.-C., B. Schmandt, and V. C. Tsai (2012). Joint inversion of Rayleigh wave phase velocity and ellipticity using USArray: Constraining velocity and density structure in the upper crust, Geophys. Res. Lett. 39, L12303, doi: 10.1029/2012GL052196.

McNutt, S. R., and T. Nishimura (2008). Volcanic tremor during eruptions: Temporal characteristics, scaling and constraints on conduit size and processes, J. Volcanol. Geoth. Res. 178, 10-18.

Metaxian, J.-P., P. Lesage, and J. Dorel (1997). Permanent tremor of Masaya Volcano, Nicaragua: Wave field analysis and source location, J. Geophys. Res. 102, 22,529-22,545.

Nakamura, Y. (1989). A method for dynamic characteristics estimation of subsurface using microtremor on the ground surface, Q. Rep. Railway Tech. Res. Inst. 30, 25-30.
Prieto, G. A., and G. C. Beroza (2008). Earthquake ground motion prediction using the ambient seismic field, Geophys. Res. Lett. 35, L14304, doi: 10.1029/2008GL034428.

Shapiro, N. M., M. Campillo, L. Stehly, and M. H. Ritzwoller (2005). High-resolution surface-wave tomography from ambient seismic noise, Science 307, 1615-1618.

Silva, W. J., S. Li, B. Darragh, and N. Gregor (1999). Surface geology based strong motion amplification factors for the San Francisco Bay and Los Angeles areas, a PEARL Rept. to PG\&E/CEC/Caltrans, Report Number SA2120-59652.

Snieder, R. (2004). Extracting the Green's function from the correlation of coda waves: A derivation based on stationary phase, Phys. Rev. E 69, $046610,1-8$.

Stutzmann, E., M. Schimmel, G. Patau, and A. Maggi (2009). Global climate imprint on seismic noise, Geochem. Geophys. Geosyst. 10, G1104, doi: 10.1029/2009GC002619.

Tsai, V. C., and G. Ekstrom (2007). Analysis of glacial earthquakes, J. Geophys. Res. 112, F03S22, doi: 10.1029/2006JF000596.

Tsai, V. C., and D. E. McNamara (2011). Quantifying the influence of sea ice on ocean microseism using observations from the Bering Sea, Alaska, Geophys. Res. Lett. 38, L22502, doi: 10.1029/2011GL049791.

Tsai, V. C., B. Minchew, M. P. Lamb, and J.-P. Ampuero (2012). A physical model for seismic noise generation from sediment transport in rivers, Geophys. Res. Lett. 39, L02404, doi: 10.1029/2011GL050255.

Wald, D. J., D. V. Helmberger, and T. H. Heaton (1991). Rupture model of the 1989 Loma Prieta earthquake from the inversion of strong-motion and broadband teleseismic data, Bull. Seismol. Soc. Am. 81, 1540-1572.

Seismological Laboratory

California Institute of Technology

Pasadena, California 91125

tsai@caltech.edu

Manuscript received 1 May 2014; Published Online 9 September 2014 